\title{
Propiedades físico-químicas y tecnológicas de la carne en dos genotipos de pollos de crecimiento lento
}

\author{
Michel, M.A.; Fernández, R.; Revidatti, F.; Sindik, M.; Sanz, P. \\ Cátedra Producción de Aves. Facultad de Ciencias Veterinarias, UNNE, Sargento Cabral 2139, \\ Corrientes (3400), Argentina. Tel/Fax: 54-379-4425753. E-mail: granja@vet.unne.edu.ar
}

\begin{abstract}
Resumen
Michel, M.A.; Revidatti, F.; Fernández, R.; Sindik, M.; Sanz, P.: Propiedades físicoquímicas y tecnológicas de la carne en dos genotipos de pollos de crecimiento lento. Rev. vet. 26: 2, 131-135, 2015. La calidad de la carne de las aves de corral es un tema complejo que puede abordarse desde diferentes perspectivas. El objetivo del trabajo fue estudiar las propiedades físico-químicas y tecnológicas de la carne proveniente de los principales cortes de pollos "Campero INTA", estableciendo diferencias atribuibles al tipo genético y sexo. Se trabajó con un lote de aves de ambos sexos conformado por las progenies de los genotipos maternos E y T (variable independiente). El análisis comparativo se realizó mediante ANOVA en un arreglo factorial, operando el genotipo y el sexo como efectos principales. La variable extracto etéreo en el corte de pechuga registró una interacción significativa sexo*genotipo $(\mathrm{F}=4,54 \mathrm{p}=0,04)$. Los valores alcanzados por los machos $\mathrm{T}$ fueron los más altos $(2,26 \pm 0,68 \%)$ y se separaron estadísticamente de los demás grupos experimentales cuyos resultados fueron $1,58 \pm 0,39 \%$ (macho E), $1,49 \pm 0,52 \%$ (hembra E) y $1,38 \pm 0,27 \%$ (hembra T). En el corte patamuslo se registró una interacción significativa sexo*genotipo en la variable pérdida por cocción a baño maría $(\mathrm{F}=4,94 \mathrm{p}=0,03)$. Los valores más altos fueron registrados por las hembras del genotipo T $(20,44 \pm 3,63 \%)$ y los más bajos por las hembras del genotipo $\mathrm{E}(14,04 \pm 5,92 \%)$, en tanto que los machos $\mathrm{E}(16,66 \pm 4,04 \%)$ y los machos $\mathrm{T}(15,86 \pm 2,82 \%)$ arrojaron valores intermedios. Se demostraron diferencias significativas por sexo para la variable pérdida por cocción en horno con valores de $34,59 \pm 6,76 \%$ (machos) y $27,22 \pm 4,52 \%$ (hembras). Se concluye que el sexo afectó a los atributos físico-químicos de la carne, registrándose un mayor contenido lipídico en los machos. Ambos genotipos tuvieron un comportamiento diferente con respecto a los atributos tecnológicos analizados, datos que amplían el conocimiento de las cualidades carniceras de la raza estudiada.
\end{abstract}

Palabras clave: pollo de crecimiento lento, genotipos, calidad de carne.

\begin{abstract}
Michel, M.A.; Revidatti, F.; Fernández, R.; Sindik, M.; Sanz, P.: Physico-chemical and technological meat properties in two genotypes of slow growth chickens. Rev. vet. 26: 2, $131-135,2015$. Meat quality in poultry is a complex area that can be approached from different perspectives. The aim of this study was to determine the physico-chemical and technological meat properties of "Campero INTA" chicken, to determine differences attributable to gender and genotype and contribute to the description of such attributes in these birds. A flock of mixed chickens with both sexes formed by the progeny of maternal genotypes $\mathrm{E}$ and $\mathrm{T}$ of this breed (independent variable) was used. A comparative analysis was performed using a factorial ANOVA with genotype and sex as main effects. Ethereal extract in breast samples showed a significant interaction sex*genotype $(\mathrm{F}=4.54 \mathrm{p}=0.04)$. Values reached by $\mathrm{T}$ males were the highest $(2.26 \pm 0.68 \%)$ and were statistically separated from another experimental groups which results were $1.58 \pm 0.39 \%$ (E male), $1.49 \pm 0.52 \%$ (E female) and $1.38 \pm 0.27 \%$ ( $\mathrm{T}$ female). In leg quarter samples it was recorded a significant interaction sex*genotype for cooking loss in double boiler $(\mathrm{F}=4.94 \mathrm{p}=0.03)$. The higher values were recorded by $\mathrm{T}$ female genotype (20.44 $\pm 3.63 \%)$ and the lowest by E female genotype (14.04 $\pm 5.92 \%)$, while E males $(16.66 \pm 4.04 \%)$ and $T$ males $(15.86 \pm 2.82 \%)$ registered intermediate values. Significant differences were observed when considering sex and cooking loss in oven variable, with values of $34.59 \pm 6.76 \%$ (male) and $27.22 \pm 4.52 \%$ (female). It can be concluded that sex affected the physical and chemical attributes of the meat, meaning this higher lipid content in males. Both
\end{abstract}


genotypes had a different behaviour with regard to the analyzed technological attributes, data that may improve the knowledge of the meat quality of the studied breed.

Key words: chicken of slow growth, genotypes, meat quality.

\section{INTRODUCCIÓN}

El pollo "Campero-INTA" desarrollado en Argentina por el Instituto Nacional de Tecnología Agropecuaria (INTA), es un ave de crecimiento lento y marcada fortaleza, cualidades que permiten su crianza en condiciones semi-extensivas o extensivas ${ }^{9}$. Sin embargo, su caracterización aún no ha sido completamente definida y diversos estudios previos han revelado diferencias atribuibles al origen genético ${ }^{14}$.

En las razas tradicionales de aves de tipo pesado y sus cruzamientos, el trabajo de mejoramiento en sus aspectos productivos aún se encuentra en desarrollo, siendo necesario adecuar las técnicas de producción a fin de que se ajusten a estos genotipos. En efecto, la mayor parte de los estudios llevados a cabo sobre las mismas han sido solo de carácter descriptivo, centrándose principalmente en las características externas ${ }^{8}$.

Por su parte, en la avicultura industrial, el ajuste constante de las técnicas de manejo que permiten el máximo aprovechamiento del potencial genético, es el enfoque más apropiado para dar solución a esta problemática. Tal idea puede ser aplicada también a los pollos de crecimiento lento, para obtener de ellos su máximo desempeño ${ }^{10,19}$. En tal sentido, es una necesidad estratégica que la tecnología producida por la ciencia avícola y utilizada en la avicultura industrial, sea incorporada a los sistemas de producción alternativos, dentro del cual se incluyen los genotipos de crecimiento lento como el que nos ocupa en esta investigación ${ }^{6}$.

La calidad de la carne de aves de corral es un área muy compleja que se puede abordar desde diferentes perspectivas. Teniendo en cuenta los intereses de la industria frigorífica y también del consumidor, los pollos deben tener altos valores de rendimiento al momento del sacrificio, acompañados de canales deseables, pero al mismo tiempo buenas características sensoriales y nutricionales ${ }^{2}$.

La cadena de producción reconoce la importancia de los atributos sensoriales de los alimentos como factor de decisión en el momento que los consumidores realizan su compra. La producción de pollos para carne en sistemas extensivos puede proporcionar atributos cárnicos determinados que son buscados por los consumidores de carne aviar. Las principales diferencias en los atributos de calidad de carne entre aves criadas en libertad y convencionalmente, están relacionados con el color, sabor y textura ${ }^{20}$. En adición, la toma de conciencia de los problemas de salud y nutricionales en la población demanda con mayor frecuencia productos originados en sistemas de producción alternativos ${ }^{19}$.

El objetivo del presente trabajo fue determinar las propiedades físico-químicas y tecnológicas de las car- nes provenientes de los principales cortes de pollos "Campero INTA", para establecer diferencias atribuibles al tipo genético y/o sexo involucrado, así como referencia para describir tales atributos en este tipo de aves.

\section{MATERIAL Y MÉTODOS}

Se trabajó con un lote de aves "Campero INTA" conformado por la progenie de dos genotipos maternos de dicho pollo (variable independiente o tratamiento). Uno de ellos, el genotipo E, es una población cerrada conformada por individuos que provienen del cruzamiento entre una raza pesada y otra semipesada, con una composición genética teórica 50\% Cornish colorada y $50 \%$ Rhode Island colorada. El otro, la población $T$, es un conjunto heterogéneo de aves que tienen en común no pertenecer a la sintética $E$, presentando un $25 \%$ de ejemplares de la población sintética CE (con una proporción teórica de 25\% Cornish, 25\% Rhode Island colorada y $50 \%$ Ross), otro $25 \%$ de ejemplares DE (con una proporción teórica de 50\% Hubbard, 25\% Cornish colorada y $25 \%$ Rhode Island colorada) y $50 \%$ ES (con una proporción teórica de 87,5\% Cornish colorada, $12,5 \%$ Rhode Island colorada).

Como genotipo paterno, para ambas poblaciones de hembras se utilizaron machos $\mathrm{AH}$, que provienen del cruzamiento entre las razas Hubbard gris y Anak.

Los ensayos experimentales fueron efectuados en la Facultad de Ciencias Veterinarias de Corrientes, Argentina, en un galpón cerrado con techo de cinc, paredes de mampostería, ventanas laterales, cielorraso de material aislante y piso de cemento alisado cubierto con cama de cáscara de arroz. De los nacimientos producidos en la semana 35 del ciclo de producción de los reproductores, se obtuvieron 140 pollitos de un día de edad con los cuales se inició el ensayo.

El interior del galpón fue dividido en 14 boxes de 2 $\mathrm{m}^{2}$ de superficie que alojaron originalmente a 10 pollos cada uno $\left(5\right.$ aves por $\left.\mathrm{m}^{2}\right)$. El agua fue provista por un bebedero de plato con recipiente invertido con capacidad de 4 litros ( $80 \mathrm{~cm}$ de perímetro) a razón de uno por compartimiento. Se utilizó un comedero tolva de 6 kilos de capacidad (125 cm de perímetro) por cada corral. Como fuente de calor para los primeros 20 días de vida se emplearon estufas eléctricas, verificando la temperatura y humedad del ambiente mediante un higrotermómetro digital.

El diseño experimental previó que en dichas instalaciones se efectuara el seguimiento del ciclo de los dos lotes de pollos (variable independiente). El ciclo de producción tuvo una duración de 84 días, y fue dividido en dos fases: inicio ( 0 a 35 días) y terminación ( 35 días a faena). 
Se utilizó un lote mixto (machos y hembras) de 140 pollos de un día de edad, provistos por la Estación Experimental del INTA Corrientes (Argentina). El lote se crió a piso en forma conjunta, recibiendo las mismas condiciones ambientales y de manejo hasta el día 35. Desde este momento fue posible separar ambos sexos de acuerdo a los caracteres sexuales externos para continuar con la fase de engorde. Se seleccionaron 21 machos y 21 hembras de cada genotipo, que se alojaron en 28 boxes de $1 \mathrm{~m}^{2}$ (14 para machos y 14 para hembras) a razón de 3 aves por compartimiento.

Finalizado el ciclo se realizó la faena, obteniéndose una muestra de carcasa por cada box, conteniendo los músculos de pechuga (pectoral superficial y supracoroideo) y muslo (tríceps femoral); la mitad de cada carcasa fue destinada al análisis físicoquímico y sobre la otra mitad se midieron las propiedades tecnológicas de los cortes, según métodos de la American Meat Science Association (AMSA) y la Association of Oficial Analytical Chemists (AOAC) ${ }^{1}$.

Para los análisis físicoquímicos, las variables dependientes fueron: $\mathrm{pH}$, agua, materia seca (estufa a $105^{\circ} \mathrm{C}$ hasta obtener peso constante) y, sobre esta última, proteínas (Kjeldahl) y extracto etéreo (Soxhlet). Como propiedades tecnológicas se evaluó el porcentaje de capacidad de retención de agua, la pérdida por cocción en horno y la pérdida por cocción en baño María.

Los análisis estadísticos incluyeron técnicas descriptivas paramétricas de cada una de las variables dependientes, ordenadas según tratamientos. La distribución de todas las variables fue constatada mediante el método de Shapiro-Wilk modificado. Se aplicó análisis de la varianza (ANOVA) para un diseño completamente al azar, evaluando las diferencias entre tratamientos de las variables dependientes, considerando límite un nivel de significancia del $5 \%{ }^{21}$, utilizando el paquete estadístico Infostat 2013.

\section{RESULTADOS}

En las tablas 1 y 2 se presenta la estadística descriptiva de los análisis físico-químicos y las propiedades tecnológicas de los cortes de pechuga y pata-muslo. Se destaca la variabilidad registrada en la mayoría de los caracteres estudiados, a excepción del pH que no mostró dicho comportamiento.
Las tablas 3 y 4 muestran los resultados obtenidos para las distintas variables empleando el sexo, el genotipo y las interacciones como fuente de variación. No se observaron diferencias significativas entre los genotipos estudiados aunque se debe destacar que el $\mathrm{pH}$ en el corte de pata-muslo arrojó una tendencia a la separación entre ambas poblaciones $(\mathrm{p}=0,09)$.

La variable extracto etéreo en el corte de pechuga registró una interacción significativa sexo*genotipo

Tabla 1. Características físico-químicas y propiedades tecnológicas de la pechuga.

Tabla 2. Características físico-químicas y tecnológicas de la pata-muslo.

\begin{tabular}{lcccccc}
\hline & $\mathrm{n}$ & $\overline{\mathrm{x}}$ & $\mathrm{DE}$ & $\mathrm{CV}$ & mín & máx \\
\hline proteína bruta (\%) & 28 & 23,15 & 3,06 & 13,24 & 18,21 & 28,46 \\
extracto etéreo (\%) & 28 & 3,54 & 1,11 & 31,34 & 1,41 & 5,48 \\
$\mathrm{pH}$ & 28 & 6,23 & 0,16 & 2,64 & 5,92 & 6,50 \\
capacidad de retención de agua (\%) & 28 & 27,04 & 4,55 & 16,81 & 19,57 & 38,03 \\
pérdidas por cocción en baño María (\%) & 28 & 16,75 & 4,69 & 27,97 & 3,00 & 23,47 \\
pérdidas por cocción en horno (\%) & 28 & 30,90 & 6,78 & 21,93 & 12,63 & 43,37 \\
\hline
\end{tabular}

\begin{tabular}{cccccc}
$\mathrm{n}$ & $\overline{\mathrm{x}}$ & $\mathrm{DE}$ & $\mathrm{CV}$ & mín & máx \\
\hline 28 & 23,57 & 3,28 & 13,91 & 18,72 & 32,45 \\
28 & 1,68 & 0,58 & 34,55 & 1,01 & 3,41 \\
28 & 5,79 & 0,13 & 2,24 & 5,55 & 6,03 \\
28 & 34,71 & 4,16 & 11,99 & 26,27 & 45,00 \\
28 & 14,54 & 6,16 & 42,36 & 5,40 & 33,44 \\
28 & 26,13 & 9,22 & 35,29 & 9,87 & 57,63 \\
\hline
\end{tabular}

estándar, min: mínimo, max: máximo. 
$(\mathrm{F}=4,54 \mathrm{p}=0,04)$. Los valores alcanzados por los machos del grupo $\mathrm{T}$ fueron los más altos $(2,26 \pm 0,68 \%)$ y se separaron estadísticamente de los demás grupos experimentales cuyos resultados fueron $1,58 \pm 0,39 \%$ (macho E), $1,49 \pm 0,52 \%$ (hembra E) y $1,38 \pm 0,27 \%$ (hembra T).

Para el corte pata-muslo se registró una interacción significativa sexo*genotipo en la variable pérdida por cocción a baño María $(\mathrm{F}=4,94 \mathrm{p}=0,03)$. Los valores más altos fueron registrados por las hembras del genotipo T $(20,44 \pm 3,63 \%)$ y los más bajos por las hembras del genotipo $\mathrm{E}(14,04 \pm 5,92 \%)$, en tanto que los machos E $(16,66 \pm 4,04 \%)$ y los machos T $(15,86 \pm 2,82 \%)$ arrojaron valores intermedios.

Se demostraron diferencias significativas por sexo para la variable pérdida por cocción en horno con valores de $34,59 \pm 6,76 \%$ (machos) y $27,22 \pm 4,52 \%$ (hembras).

\section{DISCUSIÓN}

El presente estudio tuvo como objetivo establecer diferencias en las características físico-químicas y propiedades tecnológicas en cortes de pechuga y patamuslo de los genotipos E y $\mathrm{T}$ de pollos Campero INTA, incorporando además el sexo como fuente de variación y las interacciones entre ambos factores. Se hipotetizó que existen diferencias en las variables estudiadas con base en el genotipo, por lo cual los resultados obtenidos pueden contribuir a la caracterización de estas poblaciones aviares de crecimiento lento, complementando a otros estudios que enfocan dicho proceso.

Las características físico-químicas estudiadas en este pollo no presentaron diferencias significativas según genotipos ni sexos para los cortes de pata-muslo y pechuga, a excepción del extracto etéreo. Ello coincide con estudios que señalan que los parámetros de calidad de la carne del pollo poseen una heredabilidad de moderada a alta y se encuentran marcadamente influidos por el patrimonio genético de las poblaciones ${ }^{13}$.

En ensayos análogos, trabajando en otras especies de aves, se halló una fuerte correlación genética entre la deposición de tejidos grasos y los rendimientos de las carcasas, y que todos estos caracteres fueron de mediana a alta heredabilidad ${ }^{16}$.

En estudios similares no se encontraron variaciones en los valores finales de $\mathrm{pH}$ cuando se trabajó con diferentes genotipos de pollos, observándose valores promedios entre 5,87 y 6 , los cuales coinciden con los expuestos en el presente estudio ${ }^{4,18}$. Los autores de tales investigaciones consideran que valores finales de $\mathrm{pH}$ inferiores a 5,7 afectan las propiedades tecnológicas de las carnes de pollo.

Por otro lado, en ensayos en los que se emplearon líneas genéticas seleccionadas para mayor depósito de grasa muscular en la pechuga se obtuvieron diferencias significativas para parámetros tales como peso corporal y otros relacionados a la calidad de las carcasas, incluyendo rendimiento de carcasas y de pechuga, cuando fueron comparadas con líneas comerciales tra- dicionales, con lo cual podría considerarse la posibilidad que dicho rasgo pueda ser modificado mediante la selección genética, pero solo en términos moderados ${ }^{23}$.

Reforzando dicha idea, al estudiar el efecto de las líneas genéticas sobre la composición química de la carne, se hallaron diferencias en la capacidad de digestión de los aminoácidos presentes en las materias primas, lo cual se reflejó en resultados productivos disímiles, con base en los programas de selección genética aplicados ${ }^{12}$.

Los resultados aquí obtenidos coinciden con trabajos que indican que las propiedades físico-químicas de la carne son apenas afectadas por el género cuando las aves se analizan a una misma edad de faena ${ }^{22}$, la comparación por sexos en el presente estudio solo demostró diferencias en el contenido de lípidos. Anteriores reportes aseveran que la composición lipídica de los cortes de carne en las aves es afectada por el grado de madurez ${ }^{3}$, lo cual podría explicar la mayor concentración de lípidos en los machos que en las hembras debido a que los mismos se encontraban al momento de la faena en un estado de madurez más avanzado. No obstante otros afirman que la mayor tasa de crecimiento de los machos debería resultar en valores más altos de porcentajes de proteína bruta ${ }^{11,15}$, mientras que sus cortes deberían poseer menos grasa ${ }^{22}$.

Se ha reportado que el efecto sexo puede generar variaciones en los valores finales de $\mathrm{pH}$, observándose en las hembras los datos más bajos en comparación con machos de la misma edad y genética ${ }^{15}$. Estos últimos hallazgos difieren con los resultados encontrados en este ensayo.

Del análisis de las pruebas correspondientes a las propiedades tecnológicas se desprende que los cortes de los animales de la línea $\mathrm{E}$ en general tuvieron mejor desempeño cuando se los compara con sus pares de la línea $\mathrm{T}$ y también se observó una ventaja de los machos respecto a las hembras. Al estudiar las interacciones entre ambos factores, los valores de pérdidas promedios más altos se encuentran en el grupo de hembras de la línea T, y los más bajos en los machos de la línea E. Estos resultados coinciden con publicaciones que señalan que al comparar ambos sexos, las carnes de los machos son más capaces de retener agua ${ }^{11}$, lo que se atribuye a diferencias en el tamaño de las pechuga entre machos y hembras ${ }^{7}$.

Otros autores refieren que los cortes de los machos poseen más elevados contenidos de proteína, de ahí su mayor capacidad de retener agua ${ }^{11,12}$. Sin embargo, algunas investigaciones afirman que dichas diferencias no son significativas o que las mismas no están relacionadas al sexo ${ }^{15,17}$. En el mismo sentido otros reportes tampoco mencionan haber hallado diferencias en pérdidas por cocción o goteo que puedan ser atribuidas a los genotipos, incluso cuando se compararon líneas comerciales tradicionales contra líneas de crecimiento lento ${ }^{5,7}$.

Se concluye que ambos genotipos del pollo "Campero INTA" revelaron distintos comportamientos con 
respecto a los atributos tecnológicos analizados, datos que contribuyen al mejor conocimiento de sus cualidades carniceras. Asimismo, el análisis por sexo evidenció diferencias en los atributos físico-químicos de la carne, registrándose un mayor contenido lipídico en los machos, probablemente porque a la edad del sacrificio exista una diferencia en el grado de madurez de ambos sexos.

\section{REFERENCIAS}

1. AOAC. 1990. Official methods of analysis, 15th ed.. Publ. Association of Official Analytical Chemists, Virginia (USA), $1.200 \mathrm{p}$.

2. Bogosavljevic S, Mitrovic S, Djokovic R, Doskovic V, Djermanovic V. 2010. Chemical composition of chicken meat produced in extensive indoor and free range rearing systems. Afr J Biotech 10: 9069-9075.

3. Castellini C, Mugnai C, Dal Bosco A. 2002. Meat quality of three chicken genotypes reared according to the organic system. Meat Sci 60: 219-225.

4. Corzo A, Schilling M, Loar R, Jackson V, Kin S, Radhakrishnan V. 2009. The effects of feeding distillers dried grains with solubles on broiler meat quality. Poult Sci 88: 432-439.

5. Debut M, Berri C, Baeza E, Sellier N, Arnould C, Guemené D, Jehl N, Boutten B, Jego Y, Beaumont C, LeBihan E. 2003. Variation of chicken technological meat quality in relation to genotype and preslaughter stress conditions. Poult Sci 82: 1829-1838.

6. Domecq RN. 2003. Conocimiento, competitividad y calidad de vida. Anales Curso de Post-grado sobre Desarrollo Local en la región Chaco-Corrientes (UNNE-UNGS), Argentina, p. 3-16.

7. Fanatico AC, Cavitt LC, Pillai PB, Emmert JL, Owens CM. 2005. Evaluation of slower-growing broiler genotypes grown with and without outdoor access: Meat quality. Poult Sci 84: 1785-1790.

8. Fanatico AC, Pillai PB, Emmert JL, Gbur EE, Meullenet JF, Owens CM. 2007. Sensory attributes of slowand fast-growing chicken genotypes raised indoors or with outdoor access. Poult Sci 86: 2441-2449.

9. Godínez O, García AJ, Fumero JE, Plasencia L. 2006. Comportamiento de las estirpes que dan origen al pollo campero cubano. Rev Cubana Cienc Avic 30: 113-117.

10. Jaturasitha S, Srikanchai T, Kreuzer M, Wicke $\mathbf{M}$. 2008. Differences in carcass and meat characteristics between chicken indigenous to northern Thailand (Blackboned and Thai native) and imported extensive breeds (Bresse and Rhode Island Red). Poult Sci 87: 160-169.
11. Kidd MT, Corzo A, Hoehler D, Miller ER, Dozier WA. 2005. Broiler responsiveness (Ross $\times 708$ ) to diets varying in amino acid density. Poult Sci 84: 1389-1396.

12. Kim EJ, Corzo A. 2012. Interactive effects of age, sex, and strain on apparent ileal amino acid digestibility of soybean meal and an animal by-product blend in broilers. Poult Sci 91: 908-917.

13. Le Bihan E, Millet N, Remignon H. 1999. Broiler meat quality: effect of selection for increased carcass quality and estimates of genetic parameters. Poult Sci 78: 822-826.

14. Librera JE, Di Masso RJ, Canet ZE, Font MT, Dottavio AM. 2003. Crecimiento, consumo de alimento y eficiencia alimenticia en pollos Campero INTA con diferente genotipo materno. Revista FAVE (Argentina) 2: 57-64.

15. López K, Schilling M, Corzo A. 2011. Broiler genetic strain and sex effects on meat characteristics. Poult Sci 90: 1105-1111.

16. Lotfi E, Zerehdaran S, Ahani M. 2011. Genetic evaluation of carcass composition and fat deposition in Japanese quail. Poult Sci 90: 2202-2208.

17. Mehaffey JM, Pradhan SP, Meullenet JF, Emmert JL, Mckee SR, Owens CM. 2006. Meat quality evaluation of minimally aged broiler breast fillets from five commercial genetic strains. Poult Sci 85: 902-908.

18. Schilling M, Radhakrishnan V, Thaxton Y, Christensen K. 2008. The effects of broiler catching method on breast meat quality. Meat Sci 79: 163-171.

19. Smith DP, Northcutt JK, Steinberg EL. 2012. Meat quality and sensory attributes of a conventional and a Label Rouge-type broiler strain obtained at retail. Poult Sci 91: 1489-1495.

20. Souza XR, Faria PB, Bressan MC. 2011. Proximate composition and meat quality of broilers reared under different production systems. Braz J Poult Sci 13: 15-20.

21. Steel RG, Torrie JH. 1988. Bioestadística: principios y procedimientos, McGraw-Hill/Interamericana, México, $715 \mathrm{p}$.

22. Young L, Northcutt J, Buhr R, Lyon C, Ware O. 2001. Effects of age, sex, and duration of post mortem aging on percentage yield of parts from broiler chicken carcasses. Poult Sci 80: 376-379.

23. Zhao G, Chen J, Zheng M, Wen J, Zhang Y. 2007. Correlated responses to selection for increased intramuscular fatin a chinese quality chicken line. Poult Sci 86: 23092314. 